\title{
Research Article \\ Study on the Confidence and Reliability of the Mean Seismic Probability Risk Model
}

\author{
Xiao-Lei Wang ${ }^{1,2}$ and Da-Gang $\mathrm{Lu}^{1,2,3}$ \\ ${ }^{1}$ School of Civil Engineering, Harbin Institute of Technology, Harbin 150090, China \\ ${ }^{2}$ Key Lab of Structures Dynamic Behavior and Control of China Ministry of Education, \\ Harbin Institute of Technology, Harbin 150090, China \\ ${ }^{3}$ State Key Laboratory for Disaster Reduction in Civil Engineering, Tongji University, Shanghai 200092, China
}

Correspondence should be addressed to Da-Gang Lu; ludagang@hit.edu.cn

Received 26 November 2016; Revised 26 January 2017; Accepted 30 July 2017; Published 10 October 2017

Academic Editor: Eugenijus Ušpuras

Copyright (C) 2017 Xiao-Lei Wang and Da-Gang Lu. This is an open access article distributed under the Creative Commons Attribution License, which permits unrestricted use, distribution, and reproduction in any medium, provided the original work is properly cited.

\begin{abstract}
The mean seismic probability risk model has widely been used in seismic design and safety evaluation of critical infrastructures. In this paper, the confidence levels analysis and error equations derivation of the mean seismic probability risk model are conducted. It has been found that the confidence levels and error values of the mean seismic probability risk model are changed for different sites and that the confidence levels are low and the error values are large for most sites. Meanwhile, the confidence levels of ASCE/SEI 43-05 design parameters are analyzed and the error equation of achieved performance probabilities based on ASCE/SEI 43-05 is also obtained. It is found that the confidence levels for design results obtained using ASCE/SEI 43-05 criteria are not high, which are less than 95\%, while the high confidence level of the uniform risk could not be achieved using ASCE/SEI 43-05 criteria and the error values between risk model with target confidence level and mean risk model using ASCE/SEI 43-05 criteria are large for some sites. It is suggested that the seismic risk model considering high confidence levels instead of the mean seismic probability risk model should be used in the future.
\end{abstract}

\section{Introduction}

The mean seismic probability risk model has widely been used in seismic design and safety evaluation of critical infrastructures, such as nuclear power plants. Seismic probability risk assessment is one of seismic safety evaluation methodologies for nuclear power plants [1,2]. Nowadays, the U.S. Nuclear Regulatory Commission has adopted the mean seismic probability risk model as the basis for risk-informed decision-making. ASCE/SEI 43-05, whose basis was also the mean seismic probability risk model, was a risk-consistent seismic design criterion in the United States [3]. Kennedy [4] provided the technical basis for the performance-goal based approach presented in the American Society of Civil Engineering Standard ASCE/SEI 43-05. In China, the newest modified seismic design code of nuclear power plants [5] suggested using the seismic evaluation methodology of ASCE/SEI 43-05, whose basis was also the mean seismic probability risk model. In some engineering domains other than nuclear engineering, the mean seismic risk model has also been widely studied and implemented. Lu et al. [6] conducted the seismic risk assessment for a reinforced concrete frame designed according to Chinese codes based on the mean seismic probability risk model.

However, the confidence of the mean seismic probability risk model is unknown. In other words, the mean seismic probability risk model could not convey the sense of confidence directly. Ellingwood and Kinali [7] proposed that some decision-makers may not be comfortable with the mean as a point estimation and prefer a more conservative fractile of the risk distribution, especially if consequences are severe. Many studies on the interval model of seismic risk and structural reliability have been conducted. For the 2000 SAC Federal Emergency Management Agency (FEMA) steel moment frame guidelines [8], the format based on quantitative confidence statements regarding the likelihood of the 
performance objective was provided. Lü and Yu [9] studied the interval model of seismic risk based on the mean hazard curve and capacity and demand models. Katona [10] studied the uncertainty in seismic safety analysis, including seismic probability risk assessment and seismic margin assessment, based on p-box theory. De Leon and Ang [11] conducted confidence bounds analysis on structural reliability estimations for offshore platforms, and they suggested that the estimation of percentile values, instead of mean values, of the calculated risk should be specified to ensure sufficient low risk levels for decisions-making purposes. Ang [12] proposed that the confidence level of seismic reliability model considering epistemic uncertainties should be as high as $90 \%$ to $95 \%$ for reducing the influence of epistemic uncertainties on reliability analysis results in some critical systems, while the mean safety index (obtained with the total of the combined aleatory and epistemic uncertainty) has a confidence level of only around $50 \%$. In nuclear engineering domain, high confidence of low probability of failure (HCLPF), which was defined as $95 \%$ confidence of less than $5 \%$ probability of failure and commonly used as the seismic capacity of structures, systems, and components (SSCs) of nuclear power plants, has been an important concept, which showed that SSCs of nuclear power plants should have low failure risk with high confidence facing earthquake disasters.

The mean seismic probability risk model has been accepted as a basis for risk-informed decision-making by U.S. Nuclear Regulatory Commission. However, for nuclear power plants as critical infrastructure, whose accident consequences are severe, the less failure probability risk with higher confidence should be required by decision-makers. In this paper, the confidence and error equations derivation of the approximate mean seismic probability risk are conducted. Meanwhile, the theoretical basis of ASCE/SEI 4305 code based on the approximate mean seismic probability risk model is extended to the approximate interval model. Confidence levels and error values based on these equations are then calculated. It is suggested that the seismic risk model considering high confidence levels instead of the mean probability risk model should be used for seismic design and safety evaluation of critical infrastructures such as nuclear power plants in the future.

\section{Seismic Probability Risk Models and Confidence and Error Equations}

2.1. The Approximate Point Estimation Model of Seismic Probability Risk. The limit state probability $P_{F}$ of seismic risk can be determined as the convolution of the seismic hazard curve and the fragility curve by either of the following two mathematically equivalent equations [13]:

$$
\begin{aligned}
& P_{F}=\int_{0}^{+\infty} H(a) \frac{\mathrm{d} F_{C}(a)}{\mathrm{d} a} \mathrm{~d} a \\
& P_{F}=-\int_{0}^{+\infty} F_{C}(a) \frac{\mathrm{d} H(a)}{\mathrm{d} a} \mathrm{~d} a,
\end{aligned}
$$

where $H(a)$ is the hazard curve (the annual frequency of exceeding amplitude $a$ ) and $F_{C}(a)$ is the fragility curve; (1) is equivalent to (2).

Modern seismic risk analysis, beginning with the seminal paper by Cornell [14] and some later studies by Ellingwood $[7,15]$, shows that the ground motion intensity can be represented by a type II distribution of extreme values, while the fragility function can be modelled by lognormal distribution.

The seismic hazard function approximation in closed form can be expressed as [7]

$$
H(a)=1-\exp \left[-\left(\frac{a}{u}\right)^{-K_{H}}\right] \approx\left(\frac{a}{u}\right)^{-K_{H}}=k_{I} a^{-K_{H}},
$$

where $u$ is the scale parameter, $K_{H}$ is the shape parameter, and $k_{I}$ is the constant; that is, $k_{I}=u^{K_{H}}$.

The seismic fragility can be expressed as [16-18]

$$
F_{C}(a)=\Phi\left[\frac{\ln \left(a / a_{C}\right)}{\sqrt{\beta_{R}^{2}+\beta_{U}^{2}}}\right]=\Phi\left[\frac{\ln \left(a / a_{C}\right)}{\beta_{C}}\right]
$$

in which $\Phi[]$ represents the standard normal probability integral, $a_{C}$ is the median capacity, $\beta_{R}$ and $\beta_{U}$ represent the logarithmic standard deviation in capacity of aleatory uncertainty and epistemic uncertainty, respectively, $\beta_{C}$ is the square root of the sum of the squares (SRSS) of $\beta_{R}$ and $\beta_{U}$.

Substituting (3) and (4) into (1), the so-called "risk equation" can be obtained:

$$
\begin{aligned}
& P_{F, \text { mean }}=H\left(a_{C}\right) \exp \left[\frac{\left(K_{H} \beta_{C}\right)^{2}}{2}\right] \\
& P_{F, \text { mean }}=k_{I}\left(a_{C}\right)^{-K_{H}} \exp \left[\frac{\left(K_{H} \beta_{C}\right)^{2}}{2}\right] .
\end{aligned}
$$

There are some approximations for (6): (1) the seismic hazard function, which is approximated by (3), is assumed to be linear on a log-log scale; (2) the seismic fragility function is assumed to follow the lognormal distribution.

2.2. The Approximate Interval Estimation Model of Seismic Probability Risk. The interval function of the fragility model is defined as [16-18]

$$
F_{C}(a)=\Phi\left[\frac{\ln \left(a / a_{C}\right)+\beta_{U} \Phi^{-1}(Q)}{\beta_{R}}\right],
$$

where $Q$ is the confidence parameter.

Equation (7) can be transformed to

$$
F_{C}(a)=\Phi\left[\frac{\ln \left(a /\left(a_{C} \exp \left(-\beta_{U} \Phi^{-1}(Q)\right)\right)\right)}{\beta_{R}}\right] .
$$

Equation (8) can be furtherly expressed as

$$
\begin{aligned}
F_{C}(a) & =\Phi\left[\frac{\ln \left(a / a_{C, Q}\right)}{\beta_{R}}\right] \\
a_{C, Q} & =a_{C} \exp \left(-\beta_{U} \Phi^{-1}(Q)\right) .
\end{aligned}
$$


Cornell [19] has pointed out that epistemic uncertainty can be modelled: (1) by assigning a lognormal (epistemic) distribution to $H(x)$ and (2) by an epistemic lognormal distribution on median $a_{C}$. McGuire et al. [20] suggested that, given the need to express seismic hazard with a single curve, the mean seismic hazard was the preferred single curve. In this paper, the mean seismic hazard curve $H(x)$ is used, and the epistemic uncertainty is modelled only by the lognormal distribution on the median $a_{C}$. Substituting (3) and (9) into (1), the interval estimation of the risk equation can be obtained:

$$
P_{F}(Q)=H\left(a_{C, Q}\right) \exp \left[\frac{\left(K_{H} \beta_{R}\right)^{2}}{2}\right] .
$$

Substituting (10) into (11), the risk equation considering the confidence of the fragility model can be obtained:

$$
\begin{aligned}
& P_{F}(Q) \\
& \quad=k_{I}\left[a_{C} \exp \left(-\beta_{U} \Phi^{-1}(Q)\right)\right]^{-K_{H}} \exp \left[\frac{\left(K_{H} \beta_{R}\right)^{2}}{2}\right] .
\end{aligned}
$$

Equation (12) can be furtherly expressed as

$$
\begin{aligned}
& P_{F}(Q) \\
& \quad=k_{I}\left(a_{C}\right)^{-K_{H}} \exp \left[\frac{1}{2}\left(K_{H} \beta_{R}\right)^{2}+\beta_{U} K_{H} \Phi^{-1}(Q)\right] .
\end{aligned}
$$

\subsection{The Confidence and Error Equations of the Mean Seismic} Risk Model. The mean seismic probability risk model has been widely used in seismic design and safety evaluation of some critical infrastructures, such as nuclear power plants. However, the mean seismic risk model has no direct information of confidence level, which the analyst has in the risk assessment. In order to analyze the confidence of the mean probability risk model, a new approach is proposed below in this paper.

Equation (6) represents the mean probability risk model, whose confidence level is unknown. Meanwhile, (13) is an interval risk model considering confidence level of the fragility model. The risk results could be, respectively, obtained from (6) and (13). Equation (6) represents the point estimation value of seismic risk, while (13) represents the interval estimation values of seismic risk with confidence $Q$. When $Q$ is taken as a value $Q_{\text {mean }}, P_{F}\left(Q_{\text {mean }}\right)$ obtained from (13) would be equal to $P_{F, \text { mean }}$ obtained from (6). In this paper, $Q_{\text {mean }}$ is regarded as the confidence of $P_{F \text {,mean }}$ obtained from (6). Following this idea, this paper considers that when all parameters except $Q$ of (6) and (13), which is taken as a value $Q_{\text {mean }}$, are the same, the value $Q_{\text {mean }}$ shall be regarded as the associated confidence level of the mean probability risk model. Ang [12] has discussed the confidence level of mean reliability model and also expressed the same idea with this paper. Ang [12] studied the design of an underground tunnel to resist a strong-motion earthquake and calculated the mean safety index and safety index with different confidence levels, and it was found that when the mean safety index was used in the design of the tunnel lining, the confidence for its safety was only about $50 \%$. In other words, the mean safety index was equal to a safety index with about $50 \%$ confidence level of interval safety index with different confidence levels for the given case study.

When $P_{F, \text { mean }}$ of (6) is equal to $P_{F}(Q)$ of (13) and $Q$ is taken as the value $Q_{\text {mean }}$, the equation can be expressed as

$$
\begin{gathered}
k_{I}\left(a_{C}\right)^{-K_{H}} \exp \left[\frac{\left(K_{H} \beta_{C}\right)^{2}}{2}\right]=k_{I}\left(a_{C}\right)^{-K_{H}} \\
\cdot \exp \left[\frac{1}{2}\left(K_{H} \beta_{R}\right)^{2}+\beta_{U} K_{H} \Phi^{-1}\left(Q_{\text {mean }}\right)\right] .
\end{gathered}
$$

It is assumed that all parameters of (6) and (13) except $Q$, which is taken as the value $Q_{\text {mean }}$, are the same. When neither $K_{H}$ nor $\beta_{U}$ is equal to 0 , the confidence of the mean probability risk model can be obtained as follows:

$$
Q_{\text {mean }}=\Phi\left(\frac{K_{H} \beta_{U}}{2}\right)
$$

where $K_{H}$, which represents the slope of the seismic hazard curve, can be furtherly expressed as [3]

$$
K_{H}=\frac{1}{\log \left(A_{R}\right)},
$$

where $A_{R}$ represents the ratio of spectra acceleration of probability levels $0.1 H_{D}$ and $H_{D}$, in which $H_{D}$ represents the probability of exceedance at uniform hazard response spectrum (UHRS).

From the definitions of the variables $A_{R}$ and $\beta_{U}$, we could know that $A_{R}$ is larger than 1 , so $K_{H}$ is always larger than 0 , while $\beta_{U}$ is no less than 0 . When $\beta_{U}$ is equal to 0 , which means that the perfect knowledge is obtained for the model, (15) would be not suitable, and then the confidence equation of the mean probability risk model would be furtherly expressed as

$$
Q_{\text {mean }}=100 \% \text {. }
$$

When there exists the epistemic uncertainty in the analysis or $\beta_{U}$ is larger than 0 , the confidence level of the mean probability risk model would be obtained using (15). Equation (15) shows that $Q_{\text {mean }}$, which is the confidence of the mean probability risk model, is proportional to $K_{H}$. From (15), it can be found that the confidence of the mean probability risk model in the regions with steep slopes of mean seismic hazard curves is higher than the regions with shallow slopes of the mean seismic hazard curves. So, for some critical infrastructures, such as nuclear power plants, which are located in the region with shallower slopes of mean seismic hazard curves, the mean probability risk model might be not appropriate. Typical values of $K_{H}$ in the West United States would be in the range of 3 to 6 , while in the Central and Eastern United States, $K_{H}$ is typically 2.5 or less [13]. The confidence of the mean seismic risk model for the West United States and Central and Eastern United States is, respectively, shown in Figures 1 and 2. The results show that 


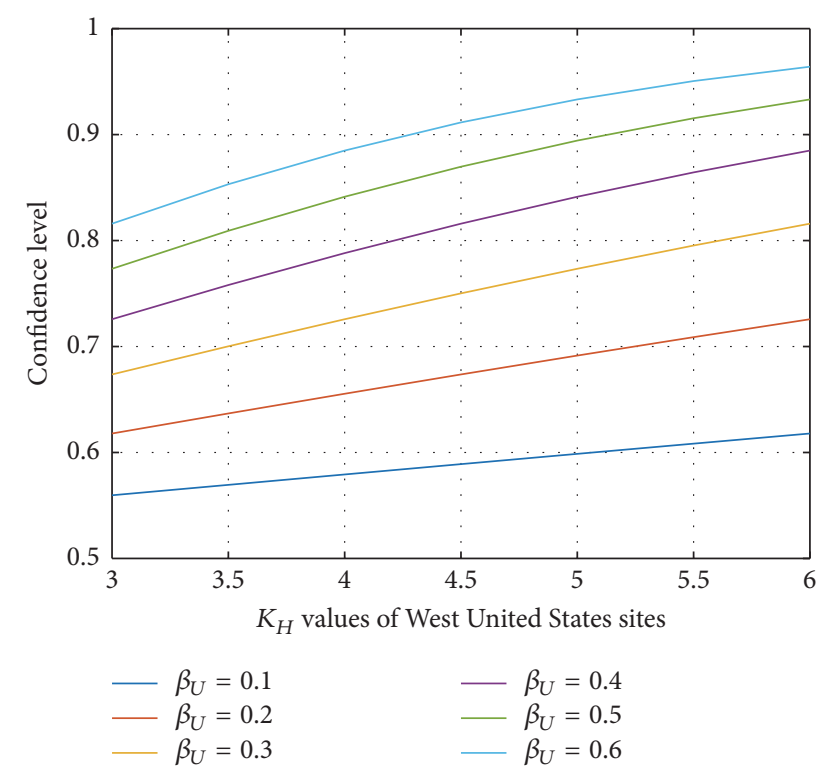

FIGURE 1: Confidence levels of mean seismic probability risk model used in West United States sites.

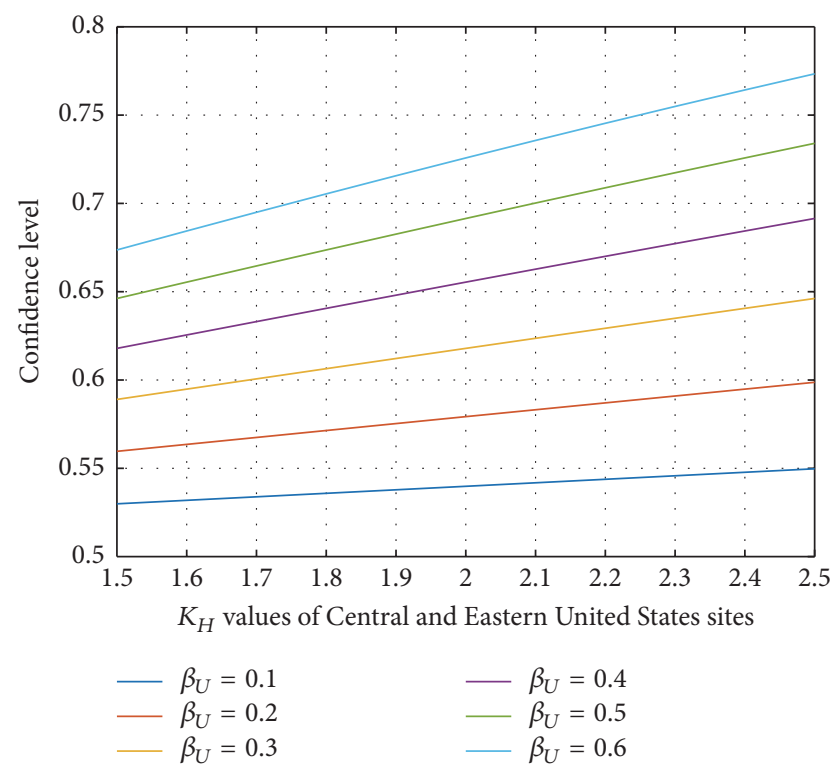

FIgURE 2: Confidence levels of mean seismic risk probability model used in Central and Eastern United States sites.

for the West United States the confidence of the mean seismic hazard model is in the range of nearly $55 \%$ to $96 \%$, while for the Central and Eastern United States the confidence of the mean seismic hazard model is in the range of nearly $52 \%$ to $78 \%$. So, when seismic risk is conducted in the Central and Eastern United States, the reliability of risk results based on the mean seismic risk model should be carefully examined and validated because of lower confidence levels.

The mean seismic risk model is obtained through calculating the mean of the distribution representing an epistemic uncertainty with the range of confidence levels. From (15) and (17), it is found that the confidence levels of the mean seismic risk model are always larger than 50\%. The physical interpretation of this phenomenon could be expressed as follows: the mean seismic risk model is more sensitive to more severe alternative models considered in the analysis; in other words, the mean seismic risk model is more sensitive to the alternative models with higher confidence levels considered in the analysis.

The confidence levels of the mean probability risk model are calculated using the parameters $\beta_{U}$, which is usually in a range from 0.1 to 0.6 , and $A_{R}$, which is usually in a range from 1.5 to 6 . The results are listed in Table 1 . It is found that, for different values of $\beta_{U}$ and $A_{R}$, the values of the confidence $Q$ are different, so for different sites, the seismic risk probability model has different confidence levels, or it can also be said that the confidence levels of the seismic risk probability model are changed for different sites. The confidence levels of the seismic probability risk model are not high, the lowest and the highest values of which are $52.56 \%$ and $95.58 \%$, respectively. We could also find that most confidence levels of the mean seismic risk model in the usual ranges of $\beta_{U}$ and $A_{R}$ are less than $90 \%$, which is low for nuclear power plants.

In fact, the influence of epistemic uncertainty on risk results is not only related to confidence level $Q$. For fully considering the reliability of the mean seismic risk model, the error equation is provided and analyzed. The error equation, which is defined as the relative distance between $P_{F}\left(Q_{\text {target }}\right)$ and $P_{F, \text { mean }}$, is expressed as follows:

$$
\begin{aligned}
\text { Err } & =\frac{P_{F}\left(Q_{\text {target }}\right)-P_{F, \text { mean }}}{P_{F, \text { mean }}}=\frac{P_{F}\left(Q_{\text {target }}\right)}{P_{F, \text { mean }}}-1 \\
& =\exp \left[\left(\Phi^{-1}\left(Q_{\text {target }}\right)-\frac{K_{H} \beta_{U}}{2}\right) K_{H} \beta_{U}\right]-1,
\end{aligned}
$$

where $Q_{\text {target }}$ is the target confidence level; Err represents the relative error of $P_{F, \text { mean }}$ with respect to $P_{F}\left(Q_{\text {target }}\right)$.

The relative distance between $P_{F}\left(Q_{0.99}\right)$ (the target confidence level is taken as 0.99 in this paper) and $P_{F \text {,mean }}$ is listed in Table 1 for usual range of $\beta_{U}$ and $K_{H}$ values. It is found that the error between $P_{F}\left(Q_{0.99}\right)$ and $P_{F \text {,mean }}$ is related not only to the confidence level, but also to the product of $\beta_{U}$ and $K_{H}$. For some sites with large $K_{H}$, even if the confidence level is high, the error is still large. So at sites with high $K_{H}$ the mean seismic risk model should be more carefully examined and validated because of their larger error.

\section{Confidence and Error Analysis of ASCE/SEI 43-05 Seismic Risk-Consistent Design Methodology}

3.1. Theoretical Basis of ASCE/SEI 43-05 Risk-Consistent Seismic Design Methodology. The theoretical basis of ASCE/SEI 43-05 risk-consistent seismic design is as follows [13].

For implementation purposes of ASCE/SEI 43-05, the parameters in the risk equation are reformulated in terms of design terms which are familiar to engineers such as 
TABLE 1: Confidence levels and error values of mean seismic probability risk model for the usual range of $\beta_{U}$ and $K_{H}$ values.

\begin{tabular}{lccccccc}
\hline & & \multicolumn{5}{c}{ Confidence levels and error results between risk model with 99\% confidence } \\
$A_{R}$ & $K_{H}$ & $\beta_{U}=0.1$ & $\beta_{U}=0.2$ & $\beta_{U}=0.3$ & $\beta_{U}=0.4$ & $\beta_{U}=0.5$ & $\beta_{U}=0.6$ \\
\hline 1.5 & 5.6789 & $61.18 \%(2.1895)$ & $71.49 \%(6.3685)$ & $80.28 \%(11.3305)$ & $87.20 \%(13.9460)$ & $92.22 \%(12.1224)$ & $95.58 \%(7.3452)$ \\
1.75 & 4.1146 & $58.15 \%(1.3930)$ & $65.96 \%(3.8346)$ & $73.14 \%(7.2461)$ & $79.47 \%(10.8745)$ & $84.82 \%(13.4364)$ & $89.15 \%(13.8175)$ \\
2 & 3.3219 & $56.60 \%(1.0495)$ & $63.01 \%(2.7618)$ & $69.09 \%(5.1830)$ & $74.68 \%(8.1008)$ & $79.69 \%(10.9961)$ & $84.05 \%(13.1603)$ \\
2.25 & 2.8394 & $55.64 \%(0.8594)$ & $61.18 \%(2.1895)$ & $66.49 \%(4.0473)$ & $71.49 \%(6.3685)$ & $76.11 \%(8.9240)$ & $80.28 \%(11.3305)$ \\
2.5 & 2.5129 & $55.00 \%(0.7385)$ & $59.92 \%(1.8374)$ & $64.69 \%(3.3476)$ & $69.24 \%(5.2539)$ & $73.51 \%(7.4455)$ & $77.45 \%(9.7071)$ \\
2.75 & 2.2762 & $54.53 \%(0.6547)$ & $59.00 \%(1.5998)$ & $63.36 \%(2.8784)$ & $67.55 \%(4.4937)$ & $71.53 \%(6.3888)$ & $75.27 \%(8.4360)$ \\
3 & 2.0959 & $54.17 \%(0.5930)$ & $58.30 \%(1.4286)$ & $62.34 \%(2.5433)$ & $66.25 \%(3.9476)$ & $69.99 \%(5.6114)$ & $73.53 \%(7.4551)$ \\
3.25 & 1.9536 & $53.89 \%(0.5456)$ & $57.74 \%(1.2993)$ & $61.53 \%(2.2926)$ & $65.20 \%(3.5383)$ & $68.74 \%(5.0212)$ & $72.11 \%(6.6895)$ \\
3.5 & 1.8380 & $53.66 \%(0.5079)$ & $57.29 \%(1.1981)$ & $60.86 \%(2.0979)$ & $64.34 \%(3.2210)$ & $67.71 \%(4.5602)$ & $70.93 \%(6.0810)$ \\
3.75 & 1.7421 & $53.47 \%(0.4771)$ & $56.91 \%(1.1166)$ & $60.31 \%(1.9424)$ & $63.62 \%(2.9681)$ & $66.84 \%(4.1913)$ & $69.94 \%(5.5885)$ \\
4 & 1.6610 & $53.31 \%(0.4515)$ & $56.60 \%(1.0495)$ & $59.84 \%(1.8152)$ & $63.01 \%(2.7618)$ & $66.10 \%(3.8897)$ & $69.09 \%(5.1830)$ \\
4.25 & 1.5914 & $53.17 \%(0.4298)$ & $56.32 \%(0.9933)$ & $59.43 \%(1.7092)$ & $62.49 \%(2.5903)$ & $65.46 \%(3.6389)$ & $68.35 \%(4.8439)$ \\
4.5 & 1.5309 & $53.05 \%(0.4112)$ & $56.08 \%(0.9453)$ & $59.08 \%(1.6194)$ & $62.03 \%(2.4455)$ & $64.90 \%(3.4271)$ & $67.70 \%(4.5566)$ \\
4.75 & 1.4778 & $52.95 \%(0.3950)$ & $55.87 \%(0.9039)$ & $58.77 \%(1.5423)$ & $61.62 \%(2.3215)$ & $64.41 \%(3.2458)$ & $67.12 \%(4.3101)$ \\
5 & 1.4307 & $52.85 \%(0.3807)$ & $55.69 \%(0.8677)$ & $58.50 \%(1.4753)$ & $61.26 \%(2.2141)$ & $63.97 \%(3.0889)$ & $66.61 \%(4.0963)$ \\
5.25 & 1.3886 & $52.77 \%(0.3681)$ & $55.52 \%(0.8358)$ & $58.25 \%(1.4165)$ & $60.94 \%(2.1201)$ & $63.58 \%(2.9517)$ & $66.15 \%(3.9093)$ \\
5.5 & 1.3507 & $52.69 \%(0.3568)$ & $55.37 \%(0.8075)$ & $58.03 \%(1.3645)$ & $60.65 \%(2.0372)$ & $63.22 \%(2.8307)$ & $65.73 \%(3.7442)$ \\
5.75 & 1.3164 & $52.62 \%(0.3466)$ & $55.24 \%(0.7821)$ & $57.83 \%(1.3180)$ & $60.38 \%(1.9633)$ & $62.90 \%(2.7231)$ & $65.35 \%(3.5974)$ \\
6 & 1.2851 & $52.56 \%(0.3374)$ & $55.11 \%(0.7592)$ & $57.64 \%(1.2763)$ & $60.14 \%(1.8971)$ & $62.60 \%(2.6268)$ & $65.01 \%(3.4660)$ \\
\hline & & & & & &
\end{tabular}

TABLE 2: Summary of earthquake design provisions of ASCE/SEI 43-05 [3].

\begin{tabular}{lcccc}
\hline $\begin{array}{l}\text { Seismic Design } \\
\text { Category (SDC) }\end{array}$ & $\begin{array}{c}\text { Target performance } \\
\text { goal }\left(P_{F}\right)\end{array}$ & Probability ratio $\left(R_{P}\right)$ & $\begin{array}{c}\text { Hazard exceedance } \\
\text { probability }\left(H_{D}\right)\end{array}$ & $\mathrm{DF}_{1}$ \\
\hline 3 & $1 \times 10^{-4}$ & 4 & $4 \times 10^{-4}$ & 0.8 \\
4 & $4 \times 10^{-5}$ & 10 & $4 \times 10^{-4}$ & 0.40 \\
5 & $1 \times 10^{-5}$ & 10 & $1 \times 10^{-4}$ & 0.80 \\
\hline
\end{tabular}

structural engineers [13]. First, the $p$-percentile value of the mean fragility function is expressed as the design capacity:

$$
a_{P}=a_{C} \exp \left[-X_{P} \beta_{C}\right],
$$

where $X_{P}$ is the $(1-p)$ percentile of the standard normal deviate.

Substituting the value $a_{C}$ of (19) into (6), the following is obtained:

$$
P_{F, \text { mean }}=k_{I}\left(a_{P}\right)^{-K_{H}} \exp \left[\frac{\left(K_{H} \beta_{C}\right)^{2}}{2}-X_{P} K_{H} \beta_{C}\right] .
$$

It is assumed that $a_{P}$ is equal to the design response spectrum (DRS) times a seismic margin factor $F_{P}$ :

$$
a_{P}=F_{P} \times \mathrm{DRS}=F_{P} \times \mathrm{DF} \times \mathrm{UHRS},
$$

where $F_{P}$ is the factor of safety representing the conservatism introduced by the seismic design criteria such as NUREG0800, and DF is the seismic design factor that is related to the uniform hazard response spectra (UHRS):

$$
H_{D}=H(\mathrm{UHRS})=k_{I}(\mathrm{UHRS})^{-K_{H}}
$$

in which $H_{D}$ is the annual probability of exceeding the UHRS.
Substituting (21) into (20) and making use of (22), we can obtain

$$
\begin{aligned}
& P_{F, \text { mean }} \\
& \quad=H_{D}\left(F_{P} \mathrm{DF}\right)^{-K_{H}} \exp \left[\frac{\left(K_{H} \beta_{C}\right)^{2}}{2}-X_{P} K_{H} \beta_{C}\right] .
\end{aligned}
$$

Equation (23) can be transformed to

$$
\begin{aligned}
& \mathrm{DF}_{\text {mean }} \\
& \quad=\left(F_{P}\right)^{-1}\left[R_{P} \exp \left(\frac{\left(K_{H} \beta_{C}\right)^{2}}{2}-X_{P} K_{H} \beta_{C}\right)\right]^{1 / K_{H}} .
\end{aligned}
$$

The design factor equations of ASCE/SEI 43-05 are expressed as follows:

$$
\begin{aligned}
\mathrm{DF} & =\operatorname{Maximum}\left(\mathrm{DF}_{1}, \mathrm{DF}_{2}\right) \\
\mathrm{DF}_{2} & =0.6\left(A_{R}\right)^{\alpha},
\end{aligned}
$$

where $\mathrm{DF}_{1}$ and $\alpha$ are defined in Table 2, respectively, for SDCs 3-5. 
3.2. Interval Estimation Functions of ASCE/SEI 43-05 RiskConsistent Seismic Design Methodology. The mean probability risk model is the theoretical basis of ASCE/SEI 43-05 risk-consistent seismic design methodology. For analyzing the confidence of the risk results based on ASCE/SEI 43-05 design methodology, the interval risk estimation functions of ASCE/SEI 43-05 are obtained. The confidence equations of ASCE/SEI 43-05 risk-consistent seismic design are as follows.

Equation (13) represents the risk equation considering the confidence of the fragility model. Substituting the value $a_{C}$ of (19) into (13), we can obtain

$$
\begin{aligned}
& P_{F}(Q)=k_{I}\left(a_{P}\right)^{-K_{H}} \\
& \cdot \exp \left[\frac{\left(K_{H} \beta_{R}\right)^{2}}{2}-X_{P} K_{H} \beta_{C}+\beta_{U} K_{H} \Phi^{-1}(Q)\right] .
\end{aligned}
$$
obtain

Substituting (21) into (26) and making use of (22), we can

$$
\begin{aligned}
& P_{F}(Q)=H_{D}\left(F_{P} \mathrm{DF}\right)^{-K_{H}} \\
& \cdot \exp \left[\frac{\left(K_{H} \beta_{R}\right)^{2}}{2}-X_{P} K_{H} \beta_{C}+\beta_{U} K_{H} \Phi^{-1}(Q)\right] .
\end{aligned}
$$

Equation (27) can be transformed to

$$
\begin{aligned}
& \operatorname{DF}(Q)=\left(F_{P}\right)^{-1}\left[R _ { P } \operatorname { e x p } \left(\frac{\left(K_{H} \beta_{R}\right)^{2}}{2}-X_{P} K_{H} \beta_{C}\right.\right. \\
& \left.\left.\quad+\beta_{U} K_{H} \Phi^{-1}(Q)\right)\right]^{1 / K_{H}} .
\end{aligned}
$$

Equations (27) and (28) represent the functions of failure probability $P_{F}$ and design factor DF considering confidence, respectively.

Solving (27) for confidence $Q$ of the mean failure probability $P_{F \text {, mean }}$, which could be calculated using (23), the following can be obtained:

$$
\begin{aligned}
& Q_{P_{F, \text { mean }}} \\
& =\Phi\left(\frac{\ln \left(P_{F, \text { mean }} / H_{D}\left(F_{P} \mathrm{DF}\right)^{-K_{H}}\right)-\left(K_{H} \beta_{R}\right)^{2} / 2+X_{P} K_{H} \beta_{C}}{\beta_{U} K_{H}}\right) .
\end{aligned}
$$

Substituting (23) into (29), we could furtherly obtain

$$
Q_{P_{F, \text { mean }}}=\Phi\left(\frac{K_{H} \beta_{U}}{2}\right)
$$

where neither $K_{H}$ nor $\beta_{U}$ is equal to 0 . According to the definitions of $K_{H}$ and $\beta_{U}, K_{H}$ is positive, while $\beta_{U}$ is no less than 0 . When $\beta_{U}$ is equal to 0 , (30) would not be suitable, so the confidence equation of $P_{F}$ could be furtherly expressed as follows:

$$
Q_{P_{F, \text { mean }}}=100 \% \text {. }
$$

Solving (28) for confidence $Q$ of the mean design factor $\mathrm{DF}_{\text {mean }}$, which could be calculated using (24), the following can be obtained:

$$
Q_{\mathrm{DF}_{\text {mean }}}=\Phi\left(\frac{\ln \left(\left(\mathrm{DF}_{\text {mean }} /\left(F_{P}\right)^{-1}\right)^{K_{H}} / R_{P}\right)-\left(K_{H} \beta_{R}\right)^{2} / 2+X_{P} K_{H} \beta_{C}}{\beta_{U} K_{H}}\right) .
$$

Substituting (24) into (32), we could furtherly obtain

$$
Q_{\mathrm{DF}_{\text {mean }}}=\Phi\left(\frac{K_{H} \beta_{U}}{2}\right)
$$

where neither $K_{H}$ nor $\beta_{U}$ is equal to 0 . According to the definitions of $K_{H}$ and $\beta_{U}, K_{H}$ is positive, while $\beta_{U}$ is no less than 0 . When $\beta_{U}$ is equal to 0 , (33) would not be suitable, so the confidence equation of DF could be furtherly expressed as

$$
Q_{\mathrm{DF}_{\text {mean }}}=100 \% \text {. }
$$

When neither $K_{H}$ nor $\beta_{U}$ is equal to 0 , the confidence functions of $P_{F}$ and DF of ASCE/SEI 43-05 could, respectively, be expressed as (30) and (33). It is found that the confidence levels of the two parameters are proportional to $K_{H}$ and $\beta_{U}$, which are the same as the confidence levels of the mean seismic risk model. This conclusion could be easily understood, as the mean seismic probability risk model is the theoretical basis of ASCE/SEI 43-05.

For three SDCs of ASCE/SEI 43-05 listed in Table 2 [3], the exact design factor (DF) and the confidence of DF could be, respectively, calculated using (24) and (33). When the exact design factor (DF) is calculated, $\beta_{C}$ is usually taken as the range of 0.3 to 0.6 , as $\beta_{C}$ lies in the range of 0.3 to 0.6 for most structures, systems, and components (SSCs) of nuclear power plants (NPPs) [13]. The DF in ASCE/SEI 43-05 could be calculated using (25). The confidence levels of the exact DF are shown in Figure 3. For analyzing the confidence of ASCE $\mathrm{DF}, \beta_{C}$ is still taken as the range of 0.3 to 0.6 and it is assumed that $\beta_{U}$ is equal to $\beta_{R}$ in this paper. So actually $\beta_{U}$ and $\beta_{R}$ are, respectively, taken as the range of $0.3 \times \sqrt{2} / 2$ to $0.6 \times \sqrt{2} / 2$ in Figure 3. 


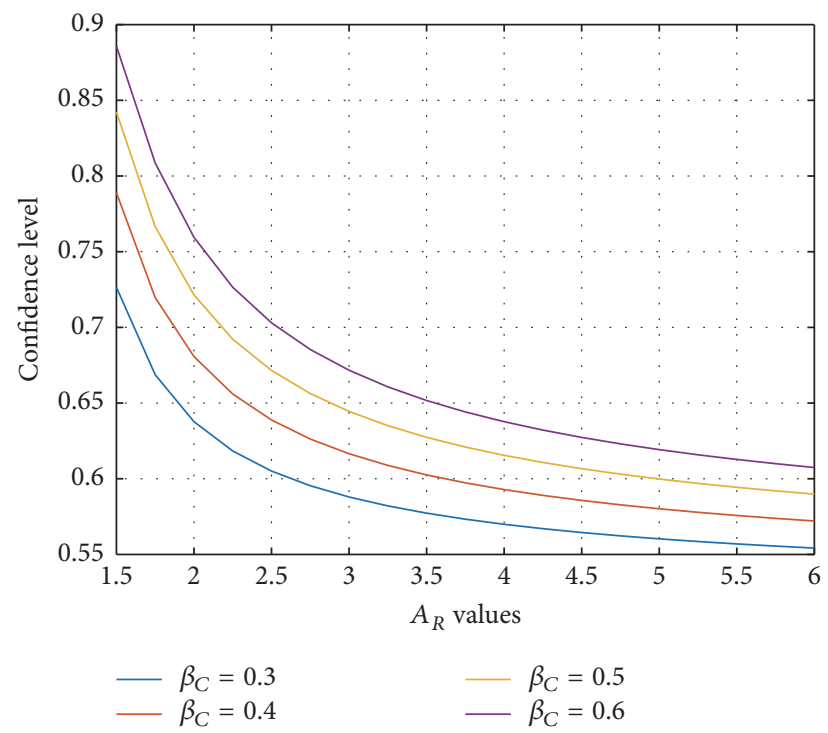

Figure 3: Confidence levels of exact DF for SDCs 3, 4, and 5 for the range of $A_{R}$ and $\beta_{C}$ values.

ASCE/SEI 43-05 [3] and Braverman et al. [13] have shown that ASCE DF approximation values approach the exact values. However, it is shown that the confidence levels of the exact DF are low, which are less than $90 \%$ from Figure 3.

For fully considering the reliability of achieved performance probabilities using ASCE/SEI 43-05 risk-consistent seismic design methodology, the error equation is obtained. The error equation, which is defined as the relative distance between point estimation function (see (23)) and interval estimation function for the target confidence level (see (27)) of achieved performance probabilities based on ASCE/SEI 43-05 risk-consistent seismic design methodology, is expressed as follows:

$$
\text { Err }=\exp \left[\left(\Phi^{-1}\left(Q_{\text {target }}\right)-\frac{K_{H} \beta_{U}}{2}\right) K_{H} \beta_{U}\right]-1
$$

It is found that the error equation of achieved performance probabilities based on ASCE/SEI 43-05 is the same as the mean seismic risk model, as the mean seismic probability risk model is the theoretical basis of ASCE/SEI 43-05.

3.3. Confidence and Error Analysis of a Design Example Based on the ASCE/SEI 43-05 Risk-Consistent Seismic Design Methodology. The typical normalized spectral acceleration hazard curves values are listed in Table 3 [3].

In ASCE/SEI 43-05, both of the following criteria need be obtained [3]:

(1) Less than $1 \%$ probability of acceptable performance for the DBE ground motion, which is defined as the Design Response Spectra (DRS)

(2) Less than $10 \%$ probability of acceptable performance for a ground motion equal to $150 \%$ of the DBE ground motion, which is defined as the DRS.
TABLE 3: Typical normalized spectral acceleration hazard curve values of ASCE/SEI 43-05 [3].

\begin{tabular}{lcccc}
\hline & \multicolumn{2}{c}{ Eastern US } & \multicolumn{2}{c}{ California } \\
$H_{(\mathrm{SA})}$ & $1 \mathrm{~Hz}$ & $10 \mathrm{~Hz}$ & $1 \mathrm{~Hz}$ & $10 \mathrm{~Hz}$ \\
& $\mathrm{SA}$ & $\mathrm{SA}$ & $\mathrm{SA}$ & $\mathrm{SA}$ \\
\hline $5 \times 10^{-2}$ & 0.014 & 0.018 & 0.087 & 0.046 \\
$2 \times 10^{-2}$ & 0.027 & 0.034 & 0.13 & 0.072 \\
$1 \times 10^{-2}$ & 0.045 & 0.055 & 0.175 & 0.100 \\
$5 \times 10^{-3}$ & 0.07 & 0.089 & 0.236 & 0.139 \\
$2 \times 10^{-3}$ & 0.143 & 0.169 & 0.351 & 0.215 \\
$1 \times 10^{-3}$ & 0.235 & 0.275 & 0.474 & 0.334 \\
$5 \times 10^{-4}$ & 0.383 & 0.424 & 0.629 & 0.511 \\
$2 \times 10^{-4}$ & 0.681 & 0.709 & 0.814 & 0.762 \\
$1 \times 10^{-4}$ & 1.00 & 1.00 & 1.00 & 1.00 \\
$5 \times 10^{-5}$ & 1.46 & 1.41 & 1.23 & 1.22 \\
$2 \times 10^{-5}$ & 2.35 & 2.13 & 1.61 & 1.51 \\
$1 \times 10^{-5}$ & 3.27 & 2.88 & 1.89 & 1.76 \\
$5 \times 10^{-6}$ & 4.38 & 3.65 & 2.2 & 2.05 \\
$2 \times 10^{-6}$ & 6.44 & 4.62 & 2.68 & 2.42 \\
$1 \times 10^{-6}$ & 8.59 & 5.43 & 3.1 & 2.72 \\
$5 \times 10^{-7}$ & 10.34 & 6.38 & 3.58 & 3.06 \\
$2 \times 10^{-7}$ & 13.21 & 7.9 & 4.24 & 3.56 \\
$1 \times 10^{-7}$ & 15.9 & 9.28 & 4.67 & 3.84 \\
\hline & & & &
\end{tabular}

TABLE 4: Seismic margin factors for different $\beta$ values calculated by Kennedy [4].

\begin{tabular}{lccccc}
\hline$\beta$ & $F_{1 \%}$ & $F_{5 \%}$ & $F_{10 \%}$ & $F_{50 \%}$ & $F_{70 \%}$ \\
\hline 0.30 & 1.10 & 1.35 & 1.5 & 2.2 & 2.58 \\
0.40 & 1 & 1.31 & 1.52 & 2.54 & 3.13 \\
0.50 & 1 & 1.41 & 1.69 & 3.2 & 4.16 \\
0.60 & 1 & 1.5 & 1.87 & 4.04 & 5.53 \\
\hline
\end{tabular}

For the two criteria in ASCE/SEI 43-05, we could calculate the achieved performance probabilities $P_{F}$ using (23) and the confidence levels of the achieved performance probabilities $P_{F}$ using (30). For different SDCs, the values of $H_{D}$ are listed in Table 2. $A_{R}$ can be obtained from Table 3, while $K_{H}$ can be calculated using $(16) . X_{p}$ means $(1-p)$ percentile of the standard normal deviate (when $p$ is equal to $1 \%, X_{p}$ is 2.326 , and when $\left.p=10 \%, X_{p}=1.282\right)$. The seismic margin factors $F_{p}$ for different $\beta$ values are calculated by Kennedy [4], as listed in Table 4.

When the achieved performance probability $P_{F}$ is calculated, $\beta_{C}$ is usually taken as the range of 0.3 to 0.6 , as $\beta_{C}$ lies in the range of 0.3 to 0.6 for most structures, systems, and components (SSCs) of nuclear power plants (NPPs) [13]. Kennedy [4] compared the results from the approximation risk equation with the precise convolution risk results, and it was found that the use of approximate power law hazard curve could give acceptable risk results. It could also be found that the failure probability designed according to ASCE/SEI 43-05 can meet two criteria $[3,4,13]$. However, it is shown that the confidence levels of failure probability $P_{F}$ are changed for different sites from Figures 4 and 5. The confidence 
TABLE 5: Error results for both the first and second criteria of ASCE/SEI 43-05 for representative hazard curves.

\begin{tabular}{|c|c|c|c|c|c|}
\hline \multirow{3}{*}{ SDC } & \multirow{3}{*}{ Hazard curves } & \multicolumn{4}{|c|}{ Error results between risk model with $99 \%$ confidence and mean risk model } \\
\hline & & $\beta_{C}=0.3$ & $\beta_{C}=0.4$ & $\beta_{C}=0.5$ & $\beta_{C}=0.6$ \\
\hline & & $F_{1 \%}=1.1$ & $F_{1 \%}=1.0$ & $F_{1 \%}=1.0$ & $F_{1 \%}=1.0$ \\
\hline \multirow{4}{*}{3} & EUS $1 \mathrm{~Hz}$ & 1.2163 & 1.8022 & 2.4889 & 3.2777 \\
\hline & EUS $10 \mathrm{~Hz}$ & 1.4066 & 2.1043 & 2.9285 & 3.8777 \\
\hline & Calif $1 \mathrm{~Hz}$ & 3.1584 & 4.9483 & 7.0248 & 9.2106 \\
\hline & Calif $10 \mathrm{~Hz}$ & 2.3304 & 3.6001 & 5.1107 & 6.8066 \\
\hline \multirow{4}{*}{4} & EUS $1 \mathrm{~Hz}$ & 1.2163 & 1.8022 & 2.4889 & 3.2777 \\
\hline & EUS $10 \mathrm{~Hz}$ & 1.4066 & 2.1043 & 2.9285 & 3.8777 \\
\hline & Calif $1 \mathrm{~Hz}$ & 3.1584 & 4.9483 & 7.0248 & 9.2106 \\
\hline & Calif $10 \mathrm{~Hz}$ & 2.3304 & 3.6001 & 5.1107 & 6.8066 \\
\hline \multirow{4}{*}{5} & EUS $1 \mathrm{~Hz}$ & 1.3967 & 2.0885 & 2.9056 & 3.8464 \\
\hline & EUS $10 \mathrm{~Hz}$ & 1.6317 & 2.4652 & 3.4559 & 4.5957 \\
\hline & Calif $1 \mathrm{~Hz}$ & 3.4400 & 5.4025 & 7.6479 & 9.9412 \\
\hline & Calif $10 \mathrm{~Hz}$ & 4.1385 & 6.5117 & 9.1067 & 11.5157 \\
\hline
\end{tabular}

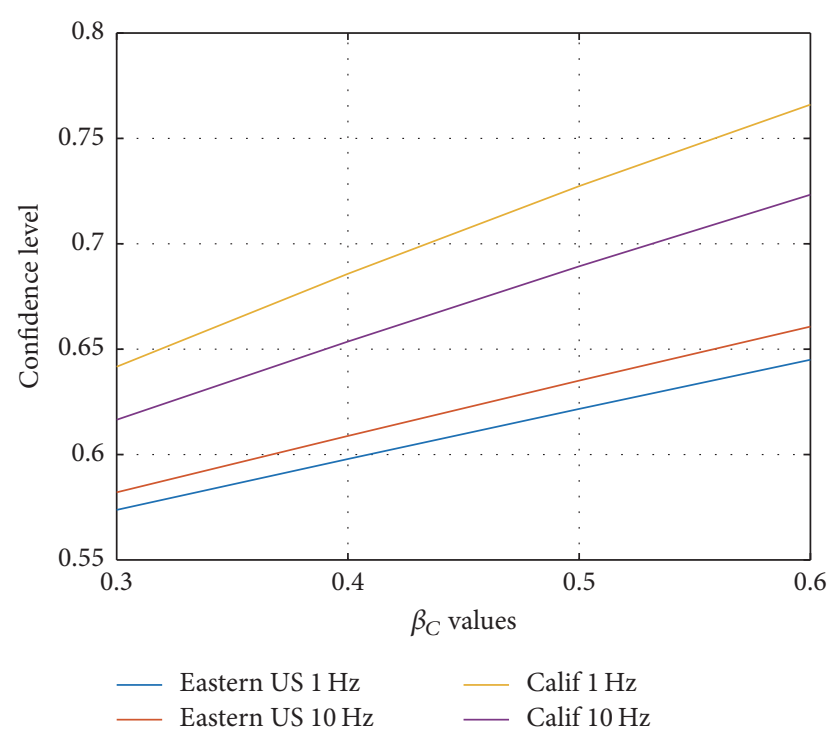

FIGURE 4: Confidence levels of achieved performance probabilities for SDCs 3 and 4 based on the first and second criteria of ASCE/SEI 43-05 for representative hazard curves.

levels of two example sites are low, which are less than $80 \%$. For analyzing the confidence of failure probability $P_{F}, \beta_{C}$ is still taken as the range of 0.3 to 0.6 and it is assumed that $\beta_{U}$ is equal to $\beta_{R}$ in this paper. So actually $\beta_{U}$ and $\beta_{R}$ are, respectively, taken as the range of $0.3 \times \sqrt{2} / 2$ to $0.6 \times \sqrt{2} / 2$ in Figures 4 and 5. We could also find that the confidence levels of the achieved performance probabilities in the region of California with steep slopes of the mean seismic hazard curves are larger than the confidence levels of the achieved performance probabilities in the regions of Eastern United States with shallow slopes of the mean seismic hazard curves. In a word, the uniform risk could be obtained using ASCE/SEI 43-05 criteria, but uniform high confidence level of the specified target risk could not be achieved based on ASCE/SEI 43-05 criteria.

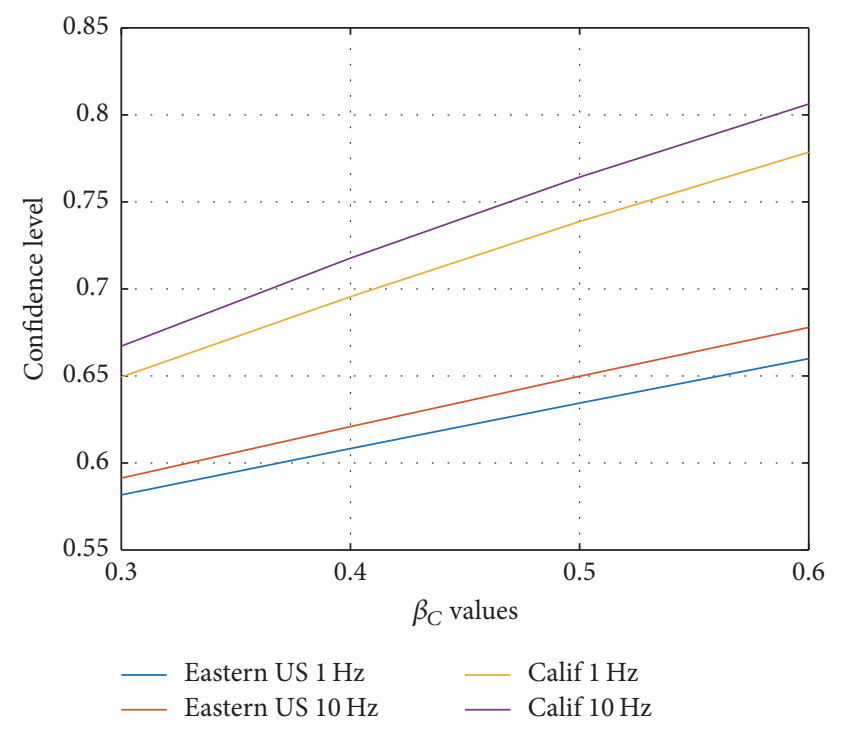

FIGURE 5: Confidence levels of achieved performance probabilities for SDC 5 based on both the first and second criteria of ASCE/SEI 43-05 for representative hazard curves.

The error analysis for achieved performance probabilities for the above example is conducted. The results are shown in Table 5. It could be found that error results between risk probabilities with target confidence level (the target confidence level is taken as 0.99 in this paper) and mean achieved performance probabilities based on ASCE/SEI 4305 are changed for different sites and the error values are large for some sites.

\section{Conclusions}

In this study, the confidence equation of the mean seismic risk model is derived. It could be found that the confidence levels of the mean probability risk model for different sites 
are changed, and the confidence levels are also low for most sites.

The error equation between $P_{F}\left(Q_{\text {target }}\right)$ and $P_{F \text {,mean }}$ is given and analyzed. It could be found that the error between $P_{F}\left(Q_{\text {target }}\right)$ (the target confidence is taken as 0.99 in the paper) and $P_{F, \text { mean }}$ is related not only to the confidence level, but also to the product of $\beta_{U}$ and $K_{H}$. So for some sites with large $K_{H}$, even if the confidence level is high, the error is still large. So at sites with high $K_{H}$ the mean seismic risk model should be more carefully examined and validated because of their larger error.

The confidence equation derivation of design factor DF and failure probability $P_{F}$ of ASCE/SEI 43-05 code are conducted. It has been found that the confidence levels of DF and $P_{F}$ of ASCE/SEI 43-05 are changed, while the confidence levels are low. It is also found that the uniform risk could be obtained, but the uniform high confidence level of the specified target risk could not be achieved using ASCE/SEI 43-05 criteria.

The error equation of achieved performance probabilities based on ASCE/SEI 43-05 risk-consistent seismic design methodology is obtained. It could be found that error results between target confidence level (the target confidence is taken as 0.99 in the paper) and achieved performance probabilities based on ASCE/SEI 43-05 are changed for different sites and the error values are large for some sites.

It is suggested that seismic risk model considering high confidence levels instead of the mean probability risk model should be used for seismic design and safety evaluation of critical infrastructures such as nuclear power plants in the future.

\section{Conflicts of Interest}

The authors declare that they have no conflicts of interest.

\section{Acknowledgments}

The financial support received from the National Science Foundation of China (Grant nos. 51678209, 51378162, and 91315301), the Research Fund of Ministry of Science and Technology of China (2013BAJ08B01), and the Open Research Fund of State Key Laboratory for Disaster Reduction in Civil Engineering (SLDRCE12-MB-04) is gratefully appreciated.

\section{References}

[1] EPRI, "A Methodology for Assessment of Nuclear Power Plant Seismic Margin,” EPRI NP-6041SL, Revision 1, EPRI, Palo Alto, Calif, USA, 1991.

[2] R. Kassawara, "Seismic Probabilistic Risk Assessment Implementation Guide," Tech. Rep., Electric Power Research Institute, 2003.

[3] ASCE, "Seismic design criteria for structures, systems, and components in nuclear facilities," ASCE/SEI 43-05, 2005.

[4] R. P. Kennedy, "Performance-goal based (risk informed) approach for establishing the SSE site specific response spectrum for future nuclear power plants," Nuclear Engineering and Design, vol. 241, no. 3, pp. 648-656, 2011.
[5] Institute of Engineering Mechanics, China Earthquake Administration. "The submitted version of the code for seismic design of nuclear power plants GB50267-97." Harbin: Institute of Engineering Mechanics China, 2012 (Chinese).

[6] D. Lu, X. Yu, M. Jia, and G. Wang, "Seismic risk assessment for a reinforced concrete frame designed according to Chinese codes," Structure and Infrastructure Engineering, vol. 10, no. 10, pp. 1295-1310, 2014.

[7] B. R. Ellingwood and K. Kinali, "Quantifying and communicating uncertainty in seismic risk assessment," Structural Safety, vol. 31, no. 2, pp. 179-187, 2009.

[8] C. A. Cornell, F. Jalayer, R. O. Hamburger, and D. A. Foutch, "Probabilistic basis for 2000 SAC federal emergency management agency steel moment frame guidelines," Journal of Structural Engineering, vol. 128, no. 4, pp. 526-533, 2002.

[9] D. Lü and X. Yu, "Theoretical study of probabilistic seismic risk assessment based on analytical functions of seismic fragility," Journal of Building Structures, vol. 34, no. 10, pp. 41-48, 2013 (Chinese).

[10] T. J. Katona, "Options for the treatment of uncertainty in seismic safety assessment of nuclear power plants," Journal of Disaster Research, vol. 8, no. 3, pp. 465-472, 2013.

[11] D. De Leon and A. H.-S. Ang, "Confidence bounds on structural reliability estimations for offshore platforms," Journal of Marine Science and Technology, vol. 13, no. 3, pp. 308-315, 2008.

[12] A. H.-S. Ang, "Target reliability for design of complex systems Role of PDEM," in Proceedings of the Symposium on Reliability of Engineering System, SRES 2015, Hangzhou, China, October 2015.

[13] J. I. Braverman, J. Xu, B. R. Ellingwood, C. J. Costantino, R. J. Morante, and C. H. Hofmayer, "Evaluation of the Seismic Design Criteria in ASCE/SEI Standard 43-05 for Application to Nuclear Power Plants," NUREG/CR-6926, BNL-NUREG77569-2007, U.S. Nuclear Regulatory Commission, 2007.

[14] C. A. Cornell, "Engineering seismic risk analysis," Bulletin of the Seismological Society of America, vol. 58, pp. 1583-1606, 1968.

[15] B. Ellingwood, "Validation studies of seismic PRAs," Nuclear Engineering and Design, vol. 123, no. 2-3, pp. 189-196, 1990.

[16] J. W. Reed and P. Kennedy R, "Methodology for Developing Seismic Fragilities," EPRI TR-103959, EPRI, Palo Alto, CA, USA, 1994.

[17] R. Campbell, G. Hardy, and K. Merz, "Seismic Fragility Application Guide," EPRI Report 1002988, EPRI, Palo Alto, CA, USA, 2002.

[18] R. Kennedy, G. Hardy, and K. Merz, "Seismic Fragility Applications Guide Update," Tech. Rep., Electric Power Research Institute, Palo Alto, CA, USA, Product ID Number 1019200, 2009.

[19] C. A. Cornell, "Risk-based structural design," in Proceedings of the Symposium on Risk Analysis, A. S. Nowak, Ed., pp. 37-48, University of Michigan, Michigan, MI, USA, 1994.

[20] R. K. McGuire, C. A. Cornell, and G. R. Toro, "The case for using mean seismic hazard," Earthquake Spectra, vol. 21, no. 3, pp. 879-886, 2005. 


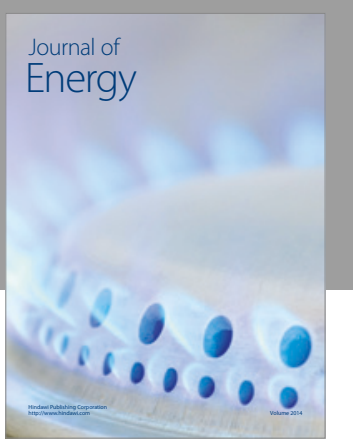

Journal of

Industrial Engineering
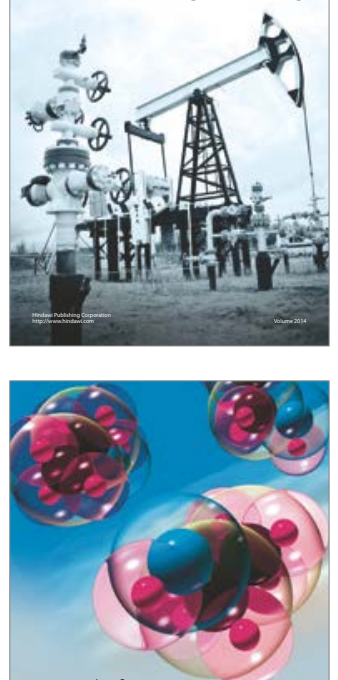

Fuels
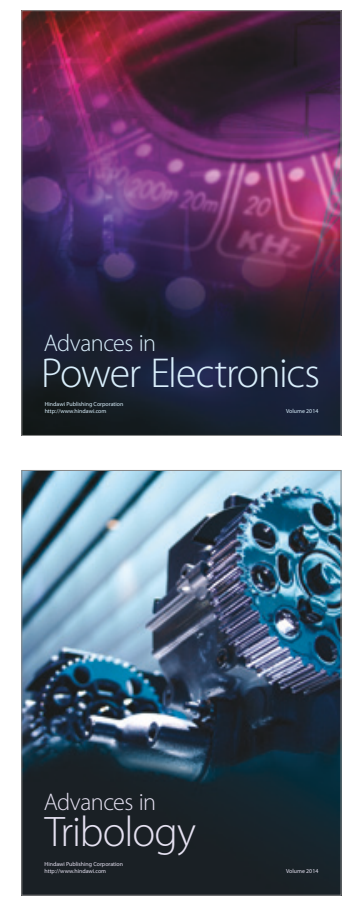
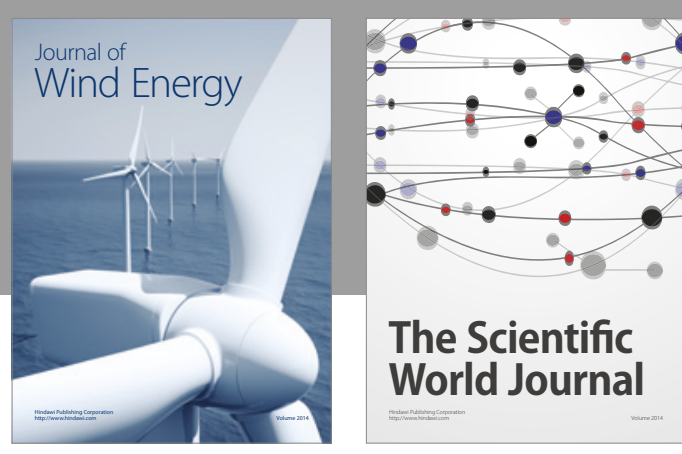

The Scientific World Journal
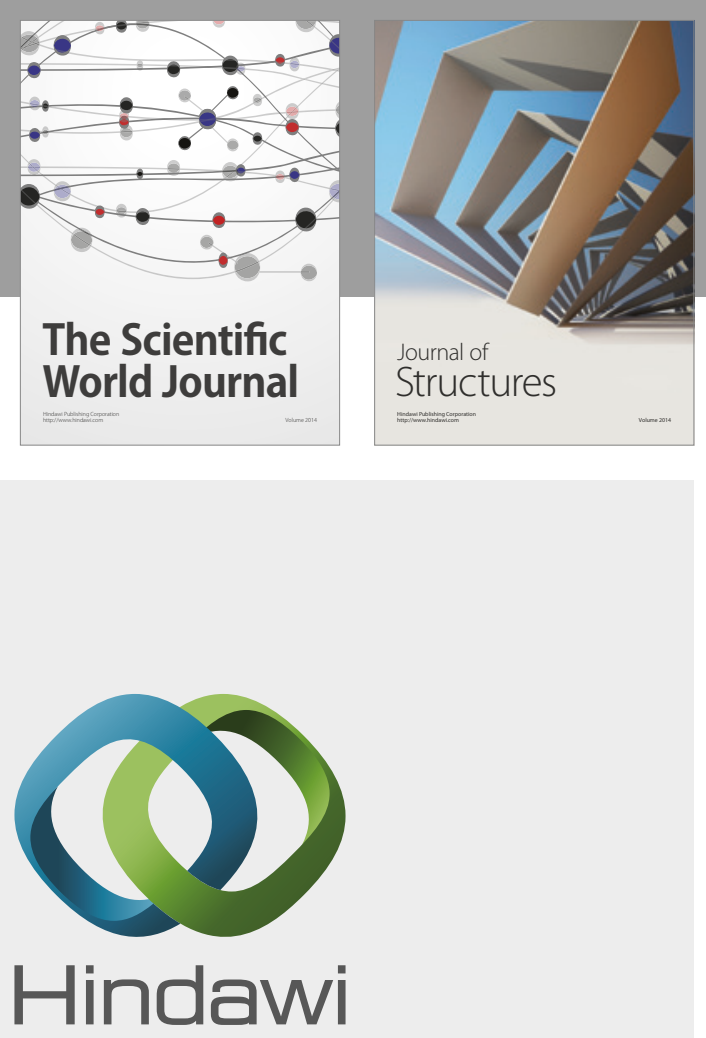

Submit your manuscripts at

https://www.hindawi.com
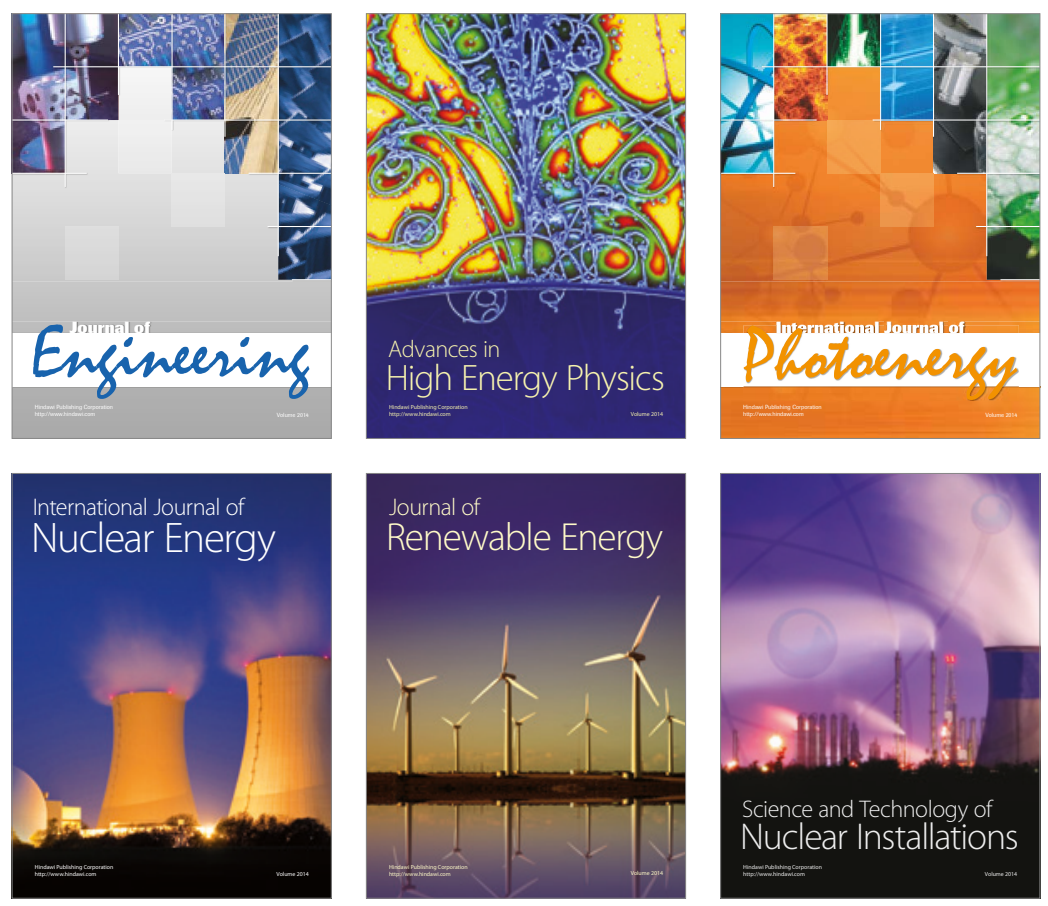

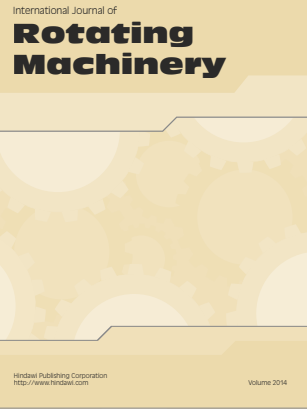

Journal of

Petroleum Engineering

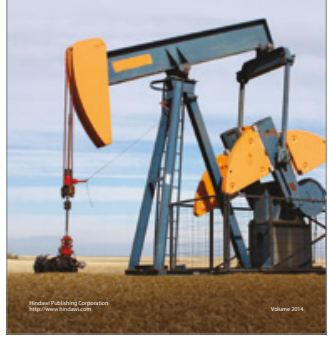

Journal of
Solar Energy
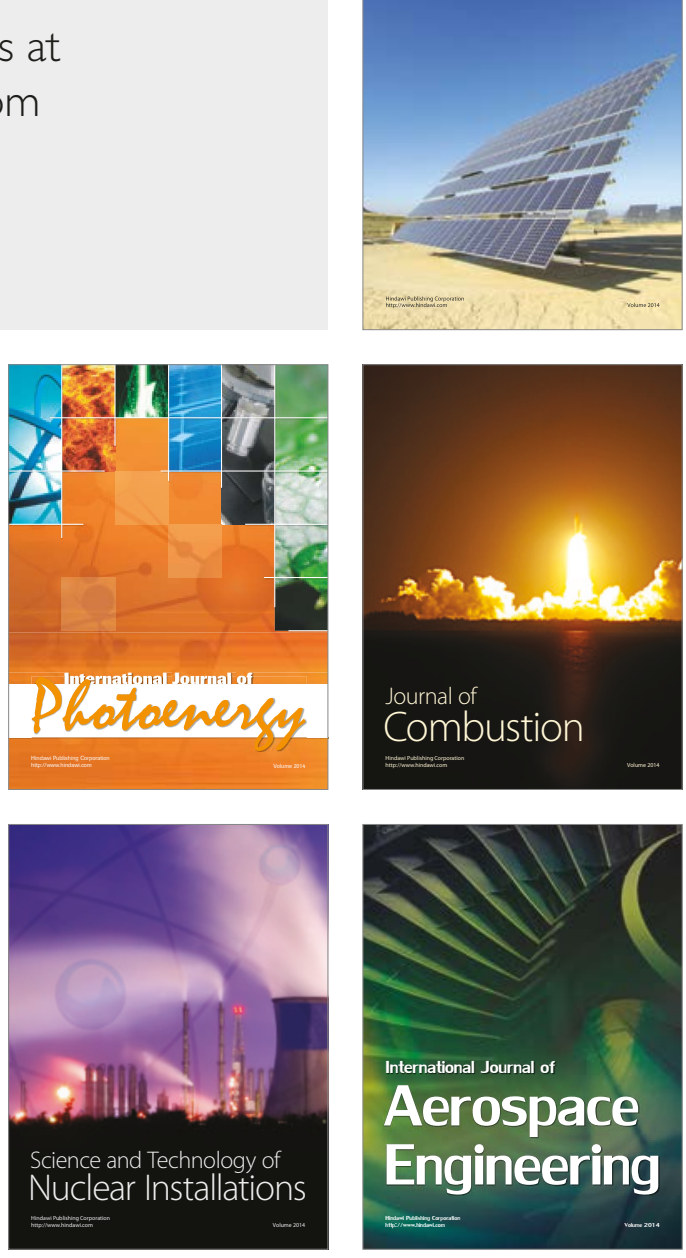